\title{
Secondary Progressive Multiple Sclerosis
}

\section{New Insights}

Bruce A.C. Cree, MD, PhD, MAS, Douglas L. Arnold, MD, Jeremy Chataway, PhD, FRCP, Tanuja Chitnis, MD, Robert J. Fox, MD, Angela Pozo Ramajo, PhD, Niamh Murphy, PhD, and Hans Lassmann, MD

Neurology ${ }^{\circledR}$ 2021;97:378-388. doi:10.1212/WNL.0000000000012323

\section{Abstract}

In most cases, multiple sclerosis (MS) begins with a relapsing-remitting course followed by insidious disability worsening that is independent from clinically apparent relapses and is termed secondary progressive MS (SMPS). Major differences exist between relapsing-remitting MS (RRMS) and SPMS, especially regarding therapeutic response to treatment. This review provides an overview of the pathology, differentiation, and challenges in the diagnosis and treatment of SPMS. We emphasize the criticality of conversion from a relapsing-remitting to a secondary progressive disease course not only because such conversion is evidence of disability progression, but also because, until recently, treatments that effectively reduced disability progression in relapsing MS were not proven to be effective in SPMS. Clear clinical, imaging, immunologic, or pathologic criteria marking the transition from RRMS to SPMS have not yet been established. Early identification of SPMS will require tools that, together with the use of appropriate treatments, may result in better long-term outcomes for the population of patients with SPMS.

\section{Introduction}

Multiple sclerosis (MS) is a chronic, inflammatory, demyelinating disease of the CNS with an unpredictable course. ${ }^{1, \mathrm{e} 1}$ Usually, MS begins with a relapsing-remitting course, in which relapses (new focal neurologic signs and symptoms caused by inflammation and demyelination) are followed by periods of remission. ${ }^{\mathrm{e} 2}$ Often, this relapsing-remitting course is followed by a phase of insidious worsening of neurologic function independent from relapses that is termed secondary progressive MS (SPMS). ${ }^{2}$ There is broad variation in the time from the first clinical manifestations of MS to the onset of SPMS; the median time from onset of relapses to evolution to SPMS is approximately 20 years (range $1-51$ years) in untreated patients. ${ }^{\text {e3 }}$

Major differences exist between relapsing-remitting MS (RRMS) and SPMS regarding responses to available treatments. ${ }^{1}$ Adapting treatment to the phase of disease is critical for patient outcomes. Until recently, no treatments with demonstrated efficacy in terms of preventing disability worsening were available for SPMS. However, siponimod was found to slow disability accumulation compared with placebo in patients with SPMS, especially in those with active disease, defined by the occurrence of relapses in the 2 years before study initiation. ${ }^{3}$ Siponimod was approved by the US Food and Drug Administration (FDA) for the treatment of relapsing forms of MS, to include clinically isolated syndrome (CIS), relapsing-remitting disease, and active secondary progressive disease, in adults, in March 2019. ${ }^{4}$ Subsequently, all disease-modifying therapies (DMTs) have also received an indication in the United States for

\author{
Correspondence \\ Dr. Cree
}

bruce.cree@ucsf.edu 


\section{Glossary}

9HPT = 9-Hole Peg Test; BBB = blood-brain barrier; BTK = Bruton tyrosine kinase $\mathbf{C D P}=$ confirmed disability progression; CIS = clinically isolated syndrome; DMT = disease-modifying therapy; EDSS = Expanded Disability Status Scale; FDA = US Food and Drug Administration; FS = Functional System; Gd = gadolinium; HR = hazard ratio; IFN = interferon; $\mathbf{M S}$ = multiple sclerosis; OR = odds ratio; PASAT = Paced Auditory Serial Addition Test; PPMS = primary progressive multiple sclerosis; RIS = radiologically isolated syndrome; RRMS = relapsing-remitting multiple sclerosis; SDMT = Symbol Digit Modalities Test; SPMS = secondary progressive multiple sclerosis; T25FW = Timed 25-Foot Walk test; TLS = tertiary lymphoid structures; TSPO $=$ translocator protein.

the treatment of active SPMS. Siponimod also received European approval in 2019 for the treatment of adults with SPMS with active disease evidenced by relapses or imaging features of inflammatory activity; this description of active disease is further detailed in the summary of product characteristics. ${ }^{5}$ In Europe, only interferon (IFN)- $\beta-1 b$ is specifically indicated in relapsing SPMS, ${ }^{6,7}$ whereas ocrelizumab and cladribine are indicated in patients with active or highly active relapsing MS (including relapsing SPMS), respectively.,

This review provides a synopsis of the pathology, differentiation, diagnosis, and treatment of SPMS, including a discussion of prognostic factors and diagnosis challenges, and a summary of recent phase 3 trials in patients with progressive forms of MS.

\section{Pathology of SPMS}

Although RRMS and SPMS are traditionally classified as distinct subtypes of MS, there is overlap in their pathologic features and disease mechanisms. ${ }^{1}$ There is an emerging view that RRMS and SPMS are part of a disease continuum with an indistinct boundary, and the underlying mechanisms driving the transition from RRMS to SPMS have not yet been wellestablished..$^{10}$ Notably, however, cortical demyelination and diffuse white matter injury appear to be involved in the transition; although these features are sparse during the early stages of RRMS, they increase with disease duration, being prominent in patients with SPMS. ${ }^{11, \mathrm{e} 4, \mathrm{e} 5}$

One theory is that MS starts primarily as an inflammatory disease (during RRMS), but later neurodegeneration independent of inflammatory responses becomes the main mechanism for disease progression (during SPMS). ${ }^{10}$ The observed between-patient differences in time elapsed between diagnosis of RRMS and diagnosis of SPMS may result from differences in functional reserve capacity in the brain. Thus, neurodegeneration is associated with progressive clinical disease in patients with preexisting brain damage exceeding their functional reserve capacity. ${ }^{\mathrm{e}}$

Brain and spinal cord inflammation is present not only in RRMS, but also in SPMS, although the extent of CNS inflammation declines with age and disease duration. ${ }^{10}$ It appears that peripheral immune responses targeting the CNS drive RRMS, whereas immune reactions within the CNS dominate SPMS. ${ }^{10}$

It has been proposed that there may be less blood-brain barrier (BBB) permeability (and, thus, impairment) in progressive MS compared with the pronounced increase in BBB permeability around the time of relapses in RRMS. ${ }^{10}$ Supporting this hypothesis, one study reported evidence of BBB damage in only $17 \%$ of long-standing lesions, with less severe damage than seen in acute lesions. ${ }^{\mathrm{e} 7}$ This compartmentalized inflammation may drive neurodegeneration and the accrual of disability seen in patients with SPMS ${ }^{\text {e8 }}$ Further studies are necessary to confirm this hypothesis. Conversely, compared with normal white matter of age-matched controls, there is a moderate increase in BBB permeability. This increase is not reflected by contrast enhancement in MRI. It is, however, associated with perivascular accumulation of fibrin irrespective of the presence or absence of inflammatory infiltrates in the brain and spinal cord lesions of patients with progressive MS. ${ }^{12, \mathrm{e} 9}$ Leakage of fibrinogen upon BBB disruption was shown to contribute to axonal damage and neurodegeneration. ${ }^{\text {e10,el1 }}$ Other pathologic processes potentially involved in neuronal cell death include iron accumulation, ${ }^{13}$ microglial activation, ${ }^{12}$ mitochondrial dysfunction, ${ }^{14}$ and oxidative stress ${ }^{15}$; these appear to be the elements of an interconnected pathogenic pathway. ${ }^{16}$

MS is historically considered a disease primarily affecting white matter of the brain. Focal white matter damage is also prominent in SPMS, and slow expansion of preexisting white matter lesions is a characteristic pathologic feature in patients with progressive disease. ${ }^{17,18, \mathrm{e} 12}$ However, the spectrum of MS pathology is broad, and different pathologies can be detected anywhere within the $\mathrm{CNS} .^{\mathrm{e} 13}$ For example, gray matter is also heavily affected, and cortical demyelination is common. The latter includes subpial, intracortical, and leukocortical lesions described in the cerebral and cerebellar cortices. $^{19, \mathrm{e} 14}$

Cortical lesions in SPMS are characterized by the absence of macrophage and leukocytic inflammatory infiltrates, and a dominant population of activated microglia. Such lesions may be driven by leptomeningeal inflammation, which is a key feature of SPMS, ${ }^{\text {e14,e15 }}$ a view supported by several studies. ${ }^{\text {e16-e18 }}$ However, it is also theoretically possible that cortical lesions drive 
meningeal inflammation. An important limitation of human histopathology studies is their cross-sectional nature. Direction of causality requires prospective assessment, which is not possible for human brain tissue studies but might be tractable with the development of new higher resolution brain MRI studies designed to interrogate the temporal relationship between meningeal tertiary follicular structures and cortical plaques.

Meningeal lymphocytic aggregates with some features of tertiary lymphoid structures have been found adjacent to large (active) subpial cortical lesions in patients with SPMS, suggesting a pathogenic role for soluble factors released from these structures. ${ }^{\text {e19 }}$ The mechanisms underlying the establishment of CNS lymphoid-like structures are not well understood. Tertiary lymphoid structures (TLS) can be found at sites of chronic inflammation, including in solid-organ tumors, in chronic inflammatory diseases, at infection sites, and in several autoimmune diseases. ${ }^{20}$ In MS, TLS are associated with widespread demyelination when present in the CNS. The effect of TLS on these various underlying disease states remains to be fully elucidated.

In progressive MS, demyelinating lesions may be equally extensive in gray and white matter; there is widespread neuroaxonal loss in white matter lesions and normal-appearing white matter, and both cortical and deep gray matter. ${ }^{21}$ Neuroaxonal loss is thought to be the principal reason for the irreversible physical and cognitive disability that occurs once patients have entered the secondary progressive stage of the disease. $^{\text {e6 }}$

Brain atrophy in MS probably reflects neuroaxonal loss and loss of myelin. Interestingly, gray matter atrophy is evident from early on in the disease course, during the RRMS phase, and spreads to involve more regions over time. ${ }^{22}$ However, in some patients with MS, there is evidence of widespread cortical atrophy early in the disease that appears to be independent of typical focal, macroscopic white matter lesions, suggesting a more diffuse, possibly neurodegenerative process. $^{\text {e20 }}$ In progressive MS, loss of brain tissue is most apparent in brain gray matter. ${ }^{\text {e21 }}$ In the spinal cord, the relationship between gray matter loss and disability is even more apparent than in the brain. ${ }^{23, \mathrm{e} 22}$

Given its key role in relaying motor and sensory signals to the cerebral cortex, the thalamus represents a critically important location in MS. ${ }^{15}$ Axonal transection within white matter lesions is thought to be a major contributor to the reduction in thalamic volume seen in MS, and thalamic atrophy may even reflect the net accumulation of MS-related damage throughout the CNS. ${ }^{15}$

In addition to MRI and histologic studies, PET studies added to the understanding of microglial activation. ${ }^{24, \mathrm{e} 23}$ PET radioligand binding to $18 \mathrm{kDa}$ translocator protein (TSPO) can be used to quantify TSPO expression. TSPO is a marker of activated microglia and macrophages and, therefore, can be used to assess innate immune activation in $\mathrm{MS}^{24,25}$ Compared with healthy controls, increased TSPO expression was observed not only in active lesions, but also in normal-appearing white matter in patients with SPMS, supporting the hypothesis that diffuse inflammation is, at least in part, due to activated microglial cells. ${ }^{26}$ In addition, increased TSPO expression is also evident in the cortex and cortical lesions, thalamus, and hippocampus in MS. TSPO expression was higher in the thalamus and hippocampus in patients with SPMS compared with patients with RRMS. This suggests a greater degree of deep gray matter neuroinflammation in SPMS. ${ }^{\text {23 }}$ In both SPMS and RRMS, increased gray matter TSPO levels correlate with disability (as measured by Expanded Disability Status Scale [EDSS] score) and, to some extent, with decline in cognitive function. ${ }^{\text {23 }}$

\section{Clinical Differentiation of SPMS From Other MS Phenotypes}

Disease relapses are the characteristic feature of RRMS and are associated with new and contrast-enhancing lesions on MRI. Gadolinium (Gd) enhancement is the hallmark of active lesions because it represents the presence of inflammatory infiltrates with leakage of the BBB. ${ }^{\mathrm{e} 13}$ Although SPMS is defined by the progressive accumulation of neurologic dysfunction in the absence of relapses, ${ }^{\mathrm{e} 2}$ evidence for disease activity may be found in patients with SPMS, who can still experience relapses. ${ }^{27}$ Furthermore, progressive forms of MS do not progress in a uniform fashion, and the disease can remain relatively stable over time. ${ }^{28}$ Therefore, classifying patients who have a progressive MS disease course (either primary progressive MS [PPMS] or SPMS) into those with or without evidence of disease activity (presence of $\mathrm{Gd}$ enhancing lesions on MRI) and those with or without evidence of disease progression within the past year (clinical evaluation) has been suggested. ${ }^{28}$

PPMS is characterized by the accrual of neurologic dysfunction and disability from disease onset in the absence of relapses that characterize RRMS, ${ }^{\mathrm{e} 15}$ although relapses may occur following PPMS onset. It remains debatable whether PPMS is a distinct entity to SPMS and RRMS. Some evidence suggests that PPMS represents a separate, noninflammatory or less inflammatory form of $\mathrm{MS}^{2}$; however, this hypothesis is challenged by pathologic findings that show profound meningeal, perivascular, and parenchymal inflammation in patients with the disease. ${ }^{1, \mathrm{e} 15, \mathrm{e} 24}$ Abundant data indicate that PPMS is a form of the disease in which acute inflammatory injury is either unrecognized clinically or does not affect eloquent brain regions that produce distinct symptoms. ${ }^{28}$ Remarkable similarities in the progressive phases of PPMS and SPMS were identified. For example, worsening proceeds at a similar rate in the 2 disease states, and the proportion of patients with superimposed relapses is similar between the two. ${ }^{\mathrm{e} 2 \mathrm{e}, \mathrm{e} 6}$ Indeed, most evidence indicates that there may be little difference between PPMS and SPMS, except for a lower incidence in the global focal white matter lesion load and, 
in particular, in the presence of Gd-enhancing lesions in PPMS. ${ }^{1}$ Conversely, the sex dimorphism that characterizes relapsingonset MS, with approximately $70 \%$ of those affected being women, is not observed in the PPMS subgroup, which is composed of equal numbers of men and women. ${ }^{\text {27 }}$

In support of the view that PPMS is not an entity distinct from SPMS and that it may represent progression after unrecognized or subclinical inflammatory injury, recent studies found that approximately $10 \%$ of patients may progress from radiologically isolated syndrome (RIS), in which lesions are observed on MRI without clinical signs or symptoms of MS, to PPMS without a clinically relapsing phase. This suggests that some individuals may go through the relapsing form of MS subclinically and that, once they reach the progressive stage, they are diagnosed with PPMS. ${ }^{29-31}$ Risk factors for development of RIS into symptomatic MS include age, positive CSF for oligoclonal bands, infratentorial lesions on MRI, and spinal cord lesions ${ }^{29,31,32}$; male sex has been identified as a risk factor in some studies ${ }^{29,31}$ but not others. ${ }^{32}$ No predictive factors for a progressive vs a relapsing clinical course following CIS have been identified. ${ }^{32}$

Although follicle-like structures are found in approximately two-thirds of patients with SPMS, ${ }^{28}$ meningeal inflammation appears to be more diffuse and uncommon in PPMS. ${ }^{10}$ This observation does not necessarily indicate that PPMS has different pathoetiology from SPMS. Instead, this discrepancy may be explained by these structures being formed during the relapsing-remitting phase of MS as a result of repeated inflammatory activity that may be a less prominent feature in PPMS. ${ }^{\text {e15 }}$ Tertiary lymphoid-like structures can be seen in a wide variety of chronic inflammatory disease states and may be related to the extent and duration of chronic inflammation. If this hypothesis is correct, these structures may better correlate with the extent and duration of relapsing disease activity and, therefore, may be more readily recognized in SPMS than in PPMS. Although PPMS remains a separate clinical entity because of the absence of exacerbations before progression, it is unlikely to be pathophysiologically distinct from SPMS. ${ }^{28}$

\section{Factors Prognostic for Evolution of SPMS}

Factors predicting progression to SPMS in patients with RRMS are not well established and, to some extent, contradictory results have been reported. ${ }^{33}$ Risk factors associated with progression to SPMS include older age at MS onset, male sex, early high relapse frequency, longer disease duration, higher baseline EDSS score, greater early increase in EDSS score, higher T2 lesion burden, spinal cord involvement, and lower brain volume. ${ }^{33-36, \mathrm{e} 29}$ In an analysis of 806 patients, the risk of entering the secondary progressive stage increased proportionally with disease duration (odds ratio [OR], 1.07 for each additional year; $p<0.001$ ), but was not influenced by the duration of the relapsing-remitting phase. ${ }^{33}$ Male sex (hazard ratio $[\mathrm{HR}], 1.41$ ), older age at onset (age $\leq 20$ years vs $>30$ years: $\mathrm{HR}, 0.52$; age $21-30$ years vs $>30$ years: HR, 0.65) independently of disease duration, and high early (during the first 2 years) relapse frequency ( $1-2$ relapses vs $\geq 3$ relapses: $H R$, 0.63 ) also predicted a significantly higher risk of SPMS (all $p<$ $0.001){ }^{33}$ It is possible that patients of an older age at disease onset have had longstanding unrecognized and untreated MS and, therefore, older age at disease onset may not be a robust prognostic factor. ${ }^{37}$ In addition to age at disease onset, current age has also been shown to increase the risk of progressing from RRMS to SPMS (OR, 1.06; $p<0.001$ ), also independently of disease duration. $^{\text {e30 }}$

Time to SPMS onset was also related to the total number of relapses during the RRMS phase, being significantly longer in patients with 5 or more attacks than in those with 1 or 2 attacks. ${ }^{33}$ However, high relapse frequency early in the disease course is predictive of shorter time to SPMS onset. ${ }^{33,38, \mathrm{e} 3, \mathrm{e} 31}$ Clinical relapses represent only the most overt consequences of inflammatory disease activity. On average, high relapse frequency equates with more inflammatory injury and hence a shorter time from disease onset to evolution of progressive disability, suggesting a link between disease activity and disease progression in untreated patients.

Patients in the original PRISMS (Prevention of Relapses and Disability by Interferon $\beta$-1a Subcutaneously in Multiple Sclerosis) randomized controlled trial of IFN vs placebo were invited to attend a single follow-up visit 15 years after initial randomization (PRISMS-15) and potential prognostic factors were analyzed. ${ }^{34}$ Of the 560 patients randomized in PRISMS, 291 returned for PRISMS-15. Change in EDSS score from baseline to 24 months was a strong predictor for conversion to SPMS at 15 years (regression coefficient, +0.5963; $p<$ $0.0001) .{ }^{34}$ Identification of additional robust markers prognostic for long-term disability could help to identify patients at higher risk of progression to SPMS. ${ }^{35}$

It is interesting to note that some patients may experience a plateau in disease progression, as evidenced by the results of an analysis of data from the population-based MS Lorraine registry, including patients over 50 years of age with no relapses for $\geq 3$ years diagnosed with relapsing MS at onset. In this population, patients who discontinued DMTs (IFN, glatiramer acetate, fingolimod, dimethyl fumarate, or teriflunomide) did not have an increased risk of relapses or an increase in EDSS scores compared with those who continued treatment. However, discontinuation was associated with a higher risk of occurrence of an EDSS score of $6 .{ }^{39}$

\section{Diagnosis of SPMS}

\section{Challenges in the Diagnosis of SPMS}

Clinical diagnosis of SPMS is challenging and tends to be retrospective, based on the patient's history of worsening after an initial relapsing disease course, ${ }^{28}$ including the patient's description of 
symptom progression. Although a standardized objective definition of SPMS has not been broadly accepted, it is typically defined as deterioration independent of relapses for $\geq 6$ months following an initial relapsing-remitting course. ${ }^{40}$ Popular opinion among physicians is that a patient needs to accumulate a minimum level of disability for a diagnosis to be made, although this is not specified in formal criteria. ${ }^{27}$ In many cases, initial symptoms of progressive MS manifest when the neurologic system is taxed, such as when walking long distances, climbing stairs, or carrying heavy objects. Occasionally, patients will report initial symptoms during aerobic exercise.

In many cases, long-term worsening takes place in patients with RRMS independently of relapses. In these patients, progression accumulates over time in early RRMS, yet a diagnosis of SPMS is not made, possibly because the loss of function happens so gradually that it remains unnoticed by patients and physicians (so-called "silent progression"). ${ }^{41,42}$ Progression independent from relapsing activity is also recognized in clinical trials of relapsing MS that quantify EDSS scores more precisely and frequently than is commonly done in observational studies or in clinical practice. ${ }^{43}$ These recent studies highlight that progression of disability can occur in typical relapsing MS and does so without association with clinical relapses, underscoring the challenge in distinguishing RRMS from SPMS.

Given that RRMS and SPMS form a continuum of disease with no clear-cut boundary between them, it is unsurprising that a period of diagnostic uncertainty, reported to last almost 3 years, often characterizes the clinical transition from RRMS to SPMS. ${ }^{40}$ A recent long-term study found that many patients with RRMS (92 of 138 with disability worsening) who had experience of confirmed clinically significant disability were still classified by their treating physicians as having RRMS instead of SPMS. Of those 92, 34 experienced disability progression independent of clinical relapses, a phenomenon termed silent progression by the investigators. ${ }^{42}$ Overall, disability worsening in this study was not associated with relapses, but all patients with disability worsening experienced accelerated brain atrophy, and this acceleration increased most in patients with silent progression. ${ }^{42}$ Nonrelapse-related sustained disability worsening that occurs early in patients with RRMS could be due to similar underlying pathology that results in disability accumulation in progressive MS. ${ }^{41,42}$

A study of 123 patients with long-term follow-up found the duration of the period of uncertainty to be $2.9 \pm 0.8$ years (mean \pm SEM) from the first visit with suggested progression to the visit at which the patient was definitively classified as having SPMS (Figure). ${ }^{40}$ A potential reason for this delay in diagnosis is that indicators of SPMS may be subtle. For example, a patient may report a worsening in their condition but the neurologic examination may detect little change in their status. ${ }^{40}$ Furthermore, many of the clinical features of MS are also observed in the normal course of aging, and it can be difficult for clinicians to determine whether findings indicate normal aging, the onset of SPMS, or a different age-related disease. ${ }^{44}$ During this diagnostic delay, patients may remain on therapies for RRMS that are ineffective for SPMS, resulting in unnecessary adverse effects and costs. $^{40}$ Once a diagnosis of SPMS has been made, levels of disease activity and progression are likely to affect therapeutic decisions. Active disease is characterized by relapses or presence of contrast-enhancing T1 hyperintense or new or enlarging T2 hyperintense lesions, as determined by MRI, whereas increasing neurologic dysfunction and disability without unequivocal recovery, independent of relapses, are indicative of progression. ${ }^{28}$

The existing definition of active SPMS, ${ }^{28}$ however, highlights a clinical challenge: if a highly effective DMT stops inflammation, the patient no longer has active MS, but disability may still accrue consistent with progression. This is perhaps the most therapeutically vexing face of MS: the progression of MS disability that occurs in the absence of active disease despite treatment with highly effective anti-inflammatory therapies. New macroscopic focal lesion formation and clinical relapses are less common in SPMS than in RRMS. Nonetheless, inflammation still occurs and is more diffuse, thus not associated with symptomatic exacerbation or contrast-enhancing MRI lesions. Diffuse inflammation could cause neuroaxonal injury and may not be efficiently targeted by some MS therapies whose mechanisms primarily involve modulation of the peripheral immune system. It is therefore possible that CNS-penetrant, anti-inflammatory medications could have clinically beneficial effects in progressive forms of MS.

In some regions of the world, regulatory approval of DMTs is restricted to RRMS. When patients transition to SPMS despite their RRMS relapses being successfully treated, they may lose eligibility for DMT, even though their relapses may return if they stop therapy. In this situation, diagnosis of SPMS could cause a patient to lose access to a (beneficial) drug, which can generate a disincentive for clinicians to diagnose SPMS. ${ }^{45}$ It is important to expedite the accurate diagnosis of SPMS to exploit the early window of opportunity for intervention in the disease and to choose the most suitable therapy or to enter appropriate clinical trials.

The availability of a treatment with proven efficacy in SPMS underscores the importance of monitoring disease progression; this will allow SPMS to be diagnosed early, enabling appropriate treatment to start in a timely manner. However, to make a definitive diagnosis, there is great reliance on patient recall of symptoms. More objective diagnostic tools are needed.

\section{Diagnostic Tools}

There is no definitive imaging or laboratory test indicative of progressive disease; however, measures of disability progression widely used in clinical practice include the EDSS, Timed 25-Foot Walk test (T25FW), and 9-Hole Peg Test (9HPT). ${ }^{\text {32 }}$ An objective evaluation of cognitive status is cognitive processing speed and that can be quantified using 


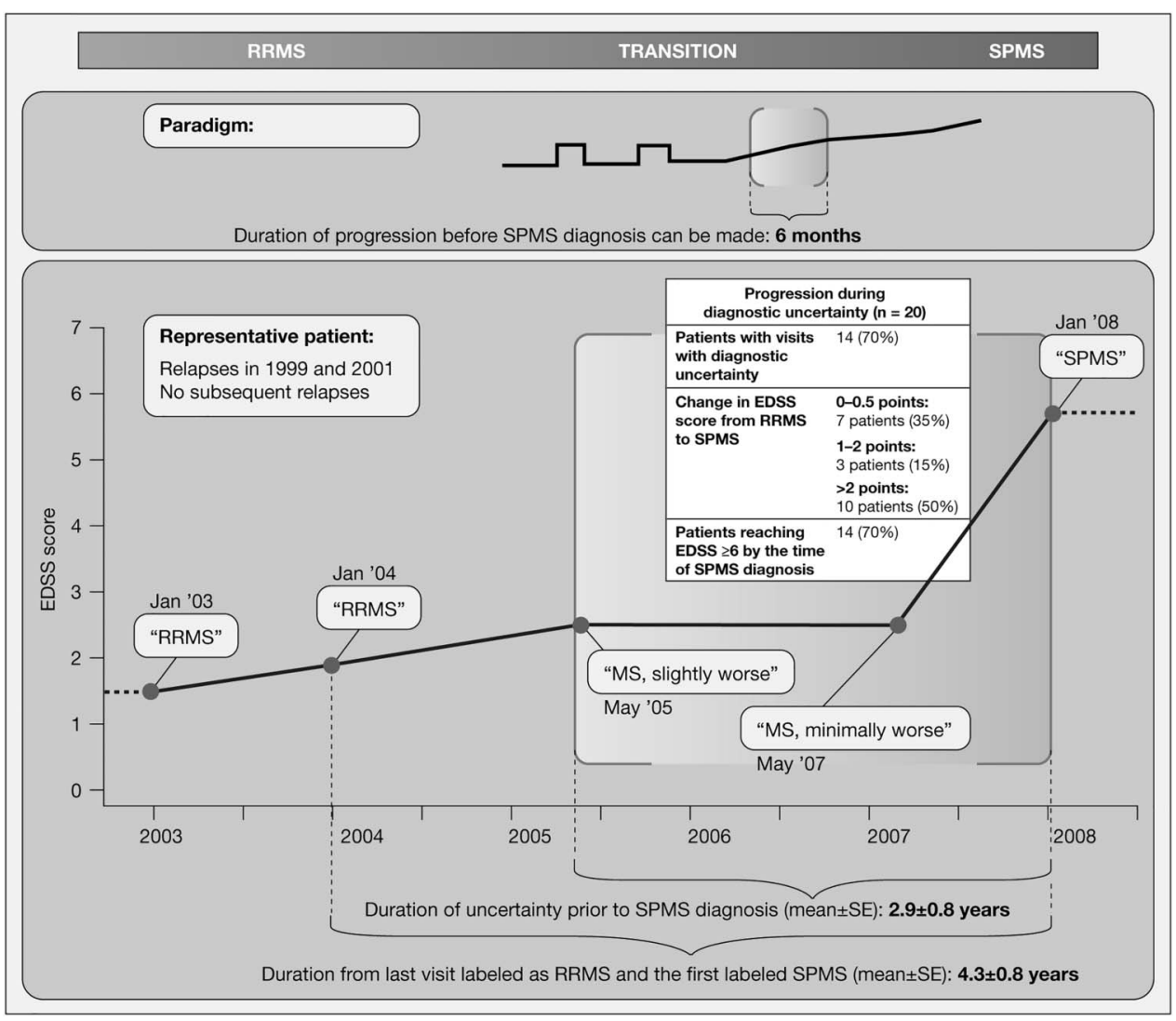

Reprinted from Katz Sand I, Krieger S, Farrell C, Miller AE. Diagnostic uncertainty during the transition to secondary progressive multiple sclerosis. Mult Scler 2014;20: 1654-1657 $7^{40}$ (copyright 2014 by SAGE Publications). Reprinted with permission of SAGE Publications, Ltd. EDSS = Expanded Disability Status Scale; $M S$ = multiple sclerosis; RRMS = relapsing-remitting multiple sclerosis; SPMS = secondary progressive multiple sclerosis. neuropsychologic tests such as the Symbol Digit Modalities Test $(\text { SDMT })^{46}$ or the Paced Auditory Serial Addition Test (PASAT) ${ }^{\text {e33 }}$ The Multiple Sclerosis Functional Composite (a combination of the T25FW, 9HPT, and PASAT-3), SDMT, or Low-Contrast Letter Acuity tests for vision can mark MS-related disability. ${ }^{46,47, \mathrm{e} 34}$ Cognitive decline is also a marker of progression, but cognitive function is not routinely assessed by neurologists in clinical practice. ${ }^{48}$ An EDSS score of 4.0 or above, which focuses on motor performance and does not assess cognitive function, ${ }^{\mathrm{e} 35}$ is frequently required for SPMS diagnosis. ${ }^{27}$ However, cognitive function can be impaired even before (or without) physical disability. ${ }^{49}$ This suggests that decline in cognitive function could be an (early) sign of progression, and an appropriate SPMS diagnosis may be missed if the EDSS score remains below 4.0 despite progression. Progressive cognitive decline related to MS is a major source of disability and loss of vocation; however, this manifestation of progressive disease is inadequately assessed by the EDSS and may require specialized testing performed by a neuropsychologist. ${ }^{\text {e36 }}$ There is superior reliability and sensitivity as well as greater patient and clinician acceptance of the SDMT than of the PASAT: the former may therefore be a superior test owing to better psychometric validity and ease of administration. ${ }^{46}$ The Brief International Cognitive Assessment for Multiple Sclerosis was developed in 2010 in an attempt to facilitate cognitive assessment and has been shown to be a relatively robust and valid measure of cognition in MS ${ }^{50}$ However, the SDMT is the only test common to all recommended cognitive batteries for patients with MS. ${ }^{46}$

Advances in mobile communications have led to the introduction of electronic health technologies, which can facilitate remote monitoring of patient symptoms. ${ }^{51}$ One study used a wrist-worn accelerometer (the commercially available Fitbit Flex; Fitbit, Inc.) to monitor step count activity in 95 patients with MS ( 35 with progressive MS) over 1 year, to assess whether average daily step count (weighted mean) is associated with other measures of MS disability. ${ }^{52}$ A significant association was seen between decreasing step count and worsening of clinical outcomes (T25FW, TimedUp-and-Go) and patient-reported outcomes (12-item Multiple Sclerosis Walking Scale). ${ }^{52}$ Interestingly, step count declined even when EDSS score remained stable, suggesting that remote monitoring using a wearable accelerometer may provide a more sensitive means of measuring disability progression than EDSS score. ${ }^{52}$ Daily physical activity, as measured by step count, was assessed as an exploratory endpoint in a large, multinational, randomized controlled trial of high-dose biotin in patients with progressive forms of MS (SPI2 [Effect of MD1003 in Progressive Multiple Sclerosis]). Other studies are investigating the use of similar devices to assess physical activity remotely, both in clinical 
trials and observational studies. Although further validation is required, in the future these tools may become integral to the long-term monitoring of disease progression in patients with MS. ${ }^{51}$

Despite the key role of biomarkers in diagnostic and therapeutic decisions in many areas of medicine, there is a distinct lack of validated biomarkers of the physiologic status or dysfunction of cells in the CNS. ${ }^{53}$ However, a proof-of-concept study published in 2017 found that it was possible to differentiate RRMS from progressive MS reliably by analyzing biomarker ratios from CSF. Twenty-one biomarker ratios distinguished RRMS from progressive MS with a validated area under the receiver operator characteristic curve of 0.91 (95\% confidence interval, $0.80-1.00) .{ }^{53}$ It was not possible, however, to differentiate SPMS from PPMS, perhaps indicating that PPMS is an equivalent disease stage to SPMS rather than a distinct noninflammatory entity. ${ }^{53}$ Although the study showed that CSF biomarkers are able to separate RRMS clearly from progressive forms of the disease, CSF biomarker-based approaches are not yet well integrated into neurology practice, and further investment will be needed to exploit their full potential. ${ }^{53}$

A refined definition of SPMS to that currently used in clinical practice may increase the sensitivity and speed of diagnosis. Using data from MSBase, including a total of 17,356 patients, investigators established a definition of SPMS based on EDSS score increasing, minimum EDSS score at progression, minimum pyramidal Functional System (FS) score at progression, time to confirmed progression, confirmation within the same FS score that triggered the progression, relapse activity before progression event, and relapse activity after progression event. ${ }^{27}$ The best definition consisted of a 1-point EDSS score increase with an EDSS score of 5.5 or less, or a 0.5-point EDSS score increase with an EDSS score of at least 6.0, in the absence of a relapse; an EDSS score of at least 4; pyramidal FS score of at least 2; and confirmed progression over at least 3 months, including confirmation of worsening within the leading $\mathrm{FS}^{27}$ The accuracy of this definition was $87 \%$ compared with the consensus opinion of 3 neurologists, and it was more sensitive, but less specific, than the physicians' diagnosis. Importantly, this new definition enabled a diagnosis of SPMS more than 3 years earlier than the diagnosis date assigned by the treating physicians. ${ }^{27}$ However, this definition likely misclassifies patients with lower EDSS scores with slow progression, who may nonetheless have progressive disease. Another SPMS diagnostic tool under development is an algorithm that integrates patient, physician, and empirical assessments. ${ }^{54}$ Multiple logistic regression analyses performed on observational study data $(\mathrm{n}=2,791)$ identified mobility (OR, 4.457; $p<0.0001)$ and self-care (OR, 2.388; $p<0.0001)$ as the strongest patientreported predictors for SPMS, and EDSS score (OR, 1.789; $p<$ $0.0001)$, age (OR, 1.037; $p<0.0001)$, and MS disease activity $(\mathrm{OR}, 1.681 ; p<0.05)$ as the most significant physicianreported predictors for SPMS. Variables of high, moderate, or low importance were integrated in the scoring algorithm, ${ }^{54}$ which requires validation for its specificity and sensitivity to correctly differentiate between RRMS and SPMS.

Table Randomized Placebo-Controlled Phase 3 Clinical Trials in Primary Progressive Multiple Sclerosis (PPMS) or Secondary Progressive Multiple Sclerosis (SPMS) Carried Out in the Past 5 Years

\begin{tabular}{|c|c|c|c|c|c|}
\hline Trial & $\begin{array}{l}\text { MS } \\
\text { type }\end{array}$ & $\mathbf{N}$ & $\begin{array}{l}\text { Study } \\
\text { length }\end{array}$ & Treatment and dosing & Key results \\
\hline $\begin{array}{l}\text { INFORMS } \\
(2016)^{60}\end{array}$ & PPMS & 970 & $\begin{array}{l}\geq 36 \text { months } \\
\text { (maximum, } \\
5 \text { years) }\end{array}$ & Fingolimod 1.25 or 0.5 mg/d & $\begin{array}{l}\text { - Fingolimod was no better than placebo in slowing disease progression } \\
\text { (composite primary endpoint based on change from baseline in EDSS } \\
\text { score, T25FW, or 9HPT scores to assess time to 3-month CDP in patients } \\
\text { treated for } \geq 3 \text { years) } \\
\text { - By the end of the study, 3-month CDP had occurred in } 232 \text { and } \\
338 \text { patients in the fingolimod and placebo groups, respectively } \\
\text { (HR, } 0.95 ; 95 \% \mathrm{Cl}, 0.80-1.12 ; p=0.544)\end{array}$ \\
\hline
\end{tabular}

\begin{tabular}{llll}
\hline $\begin{array}{l}\text { ORATORIO PPMS } 732 \geq 120 \text { weeks } \\
\text { (2017) }^{\text {337 }}\end{array}$ & $\begin{array}{l}\text { Ocrelizumab } \\
200 \mathrm{mg} \mathrm{IV} \text { every } 24 \text { weeks }\end{array}$ & $\begin{array}{l}\text { - Proportion of patients with 12-week CDP was significantly lower in the } \\
\text { ocrelizumab group than in the placebo group (32.9\% vs 39.3\%, } \\
\text { respectively; HR, 0.76; } 95 \% \mathrm{Cl}, 0.59-0.98 ; p=0.03)\end{array}$
\end{tabular}

ASCEND SPMS $889 \quad 2$ years Natalizumab Natizumab did not reduce progression on the primary

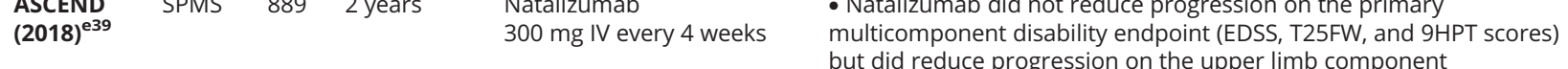
but did reduce progression on the upper limb component (9HPT; OR, 0.56; 95\% Cl, 0.40-0.80; nominal $p=0.001$ )

\begin{tabular}{|c|c|c|c|c|c|}
\hline $\begin{array}{l}\text { EXPAND } \\
(2018)\end{array}$ & SPMS & 1,651 & 3 years & Siponimod 2 mg/d & $\begin{array}{l}\text { - } 288 / 1,096(26 \%) \text { of patients receiving siponimod and } 173 / 545(32 \%) \\
\text { of patients receiving placebo had CDP at } 3 \text { months } \\
(\mathrm{HR}, 0.79 ; 95 \% \mathrm{Cl}, 0.65-0.95 \text {; relative risk reduction, } 21 \% ; p=0.013)\end{array}$ \\
\hline $\begin{array}{l}\mathrm{SPI} 2^{59} \\
(2020)\end{array}$ & $\begin{array}{l}\text { SPMS } \\
\text { or } \\
\text { PPMS }\end{array}$ & 642 & 27 months & $\begin{array}{l}\text { Existing DMT plus MD1003 } \\
\text { (biotin) } 100 \mathrm{mg} \text { tid }\end{array}$ & $\begin{array}{l}\text { - MD1003 did not improve MS-related disability (primary endpoint), } \\
\text { assessed by proportion of patients with }>20 \% \text { decrease in EDSS score } \\
\text { or T25FW at month } 12 \text {, confirmed at month } 15 ; 12.0 \% \text { of patients } \\
\text { receiving MD1003 and } 9.2 \% \text { of those receiving placebo met the primary } \\
\text { endpoint (OR, } 1.35 ; 95 \% \mathrm{Cl}, 0.81-2.26 \text { ) }\end{array}$ \\
\hline
\end{tabular}

Abbreviations: 9HPT = 9-Hole Peg Test; CDP = confirmed disease progression; $\mathrm{Cl}=$ confidence interval; DMT = disease-modifying therapy; EDSS = Expanded Disability Status Scale; HR = hazard ratio; MS = multiple sclerosis; OR = odds ratio; T25FW = Timed 25-Foot Walk test; tid = 3 times daily. 


\section{Treatment of Progressive MS: Phase 3 Trials in PPMS and SPMS}

Although many effective therapies for RRMS are available, similar success has not been seen with clinical trials of treatments for PPMS and SPMS. Phase 3 clinical trials in these patient populations published in the past 5 years are summarized in the table. In the INFORMS study in patients with PPMS (Open-label, Single-arm Extension Study [CFTY720D2306E1] to a Double-blind, Randomized Multicenter, Placebo-controlled, Parallel-group Core Study [CFTY720D2306] in PPMS), fingolimod significantly reduced inflammatory activity (measured by new lesion formation on MRI) compared with placebo but did not slow disease progression, which perhaps suggests that active disease-defined contrast-enhancing MRI lesions are not the primary mechanism of disability progression in PPMS. ${ }^{55}$ This is consistent with pathophysiologic findings suggesting that disability progression in PPMS is driven by immune reactions within the CNS leading to axonal damage and neurodegeneration, with peripherally mediated inflammation playing a less prominent role. To date, the only trial in PPMS to have met its primary efficacy endpoint (proportion of patients with 12-weeks confirmed disability progression [CDP]) is ORATORIO (A Study of Ocrelizumab in Participants With Primary Progressive Multiple Sclerosis), the results of which led to ocrelizumab (a monoclonal antibody selectively depleting CD20+ $\mathrm{B}$ cells ${ }^{56}$ ) being approved by the FDA in 2017 to treat adults with PPMS (as well as those with relapsing forms of the disease). ${ }^{57}$ Later in 2017, the European Medicines Agency approved ocrelizumab for the treatment of early PPMS with imaging features characteristic of inflammatory activity. 9.58 The efficacy of ocrelizumab in PPMS was partly attributed to its ability to quench peripherally mediated inflammation, evidenced by marked reductions in active disease-defined contrastenhancing MRI lesions, which occurred frequently in the population investigated in ORATORIO. ${ }^{\text {37,e38 }}$ Results of subsequent analyses suggested that ocrelizumab also reduced longitudinal measures of chronic lesion activity in iron-rim slowly expanding and nonslowly expanding preexisting $\mathrm{T} 2$ lesions. ${ }^{\mathrm{e} 38}$ This is consistent with focal white matter damage being an important pathologic feature in patients with progressive disease.

In ASCEND (A Clinical Study of the Efficacy of Natalizumab on Reducing Disability Progression in Participants With Secondary Progressive Multiple Sclerosis), a phase 3 trial of 2 years of duration, the effect of natalizumab compared with placebo on disability progression was investigated in patients with SPMS. Natalizumab has a strong quenching effect on peripherally mediated inflammation and is considered a highly effective DMT in patients with RRMS. However, in ASCEND, natalizumab did not reduce the proportion of patients with CDP (primary endpoint) vs placebo. ${ }^{39}$ The first trial in SPMS to have met its primary efficacy outcome in 20 years is EXPAND (Exploring the Efficacy and Safety of Siponimod in
Patients With Secondary Progressive Multiple Sclerosis), ${ }^{3}$ a phase 3 trial that evaluated the efficacy and safety of siponimod compared with placebo. In EXPAND, siponimod significantly reduced the risk of 3 -month CDP by $21 \%$ vs placebo. The positive results of EXPAND led to siponimod being approved as the first treatment specifically indicated for patients with active SPMS. ${ }^{5}$ Evidence from clinical and preclinical studies suggests that siponimod has a dual mechanism of action, with effects on both peripherally mediated inflammation as well as direct anti-inflammatory and promyelination effects on the CNS. The former likely drive the reductions in disease activity seen in EXPAND, ${ }^{3}$ while the latter drive the slowing of gray matter atrophy and promyelination effects of siponimod. ${ }^{\mathrm{e} 40, \mathrm{e} 41}$ The findings from ASCEND and EXPAND are again consistent with disability in progressive forms of MS being driven by immune reactions within the CNS leading to neurodegeneration, with peripherally mediated inflammation playing a less prominent role. Therefore, for a DMT to be effective, it needs to be able to target compartmentalized CNS inflammation in addition to peripherally mediated inflammation. Recently, the results of a 27-month phase 3 study (the SPI2 study) evaluating the efficacy and safety of MD1003 (biotin) added to existing DMT compared with placebo in patients with nonactive progressive MS (PPMS or SPMS) have been published. Neither the primary endpoint (improvement of MS-related disability) nor the secondary endpoints (including prevention of disability worsening measured by the EDSS) were met, ${ }^{59}$ suggesting that any potential effects of MD1003 (biotin) on myelin repair are not sufficient to observe beneficial effects on neurodegeneration in this patient population.

As with all randomized clinical trials, it is not possible to know whether the findings under the controlled conditions of the trial will be generalizable owing to use of inclusion and exclusion criteria designed to define a participant cohort most likely to yield interpretable results and to be free of other potentially confounding health conditions. Therefore, postmarketing studies are needed to corroborate the findings of phase 3 trials in patients with progressive MS to determine whether the benefits and risks of an intervention are similar in patients whose demographics (e.g., age) and comorbidities (e.g., history of cancer) would have resulted in exclusion from the clinical trials leading to approval of a particular product.

\section{Expert Opinion and Future Directions}

Multiple attempts have been made at defining SPMS clinically. However, a standardized definition that is broadly accepted does not exist. A common limitation of the existing definitions is reliance on EDSS to assess disability progression, although the limitations of this scale, including a strong bias towards ambulation, are widely recognized. Use of the EDSS in diagnosing SPMS leads to a tautology in which SPMS is defined by ambulatory dysfunction because much of the EDSS is defined by ambulation. Definitions based on EDSS tend to have good specificity, meaning that most patients diagnosed truly have 
progressive disease, but poor sensitivity, failing to identify patients in whom progression is driven by functional domains that are not as adequately assessed by EDSS, most notably cognition and fatigue. Therefore, a more thorough assessment of patients with SPMS that takes into account other manifestations of MS disability is needed. Recently, several tools were developed to facilitate patient-physician discussions of MS progression by structured capturing of a patient's clinical history in terms of MS symptoms and their effect on daily activities as well as cognitive function. ${ }^{54, \mathrm{e} 42}$ Once fully validated, standardized qualitative assessment of progression using such tools may reduce diagnostic uncertainty.

Multiple prognostic factors for progression have been proposed. A common limitation of natural history studies investigating prognostic factors is that data are usually obtained both prospectively and retrospectively, which can introduce information bias and could explain why some contradictory results have been reported. Furthermore, natural history studies are subject to other potential sources of bias, such as attrition bias (participants lost to follow-up may be systematically different from those retained in the study), selection bias (MS natural history studies are rarely population based), information bias (systematic distortion in data collection for either risk factors or outcome variables), response bias (systematic deviation in participant reporting), as well as confounding by unknown or unidentified variables. Lastly, MRI data are frequently absent in natural history studies. Currently, many MRI markers with potential prognostic value for progression are being investigated. Among these, gray matter atrophy and spinal cord damage have emerged as the most promising, with high specificity and sensitivity to detect progression. ${ }^{\mathrm{e} 3}$ A promising emerging prognostic MRI marker for progression is the presence of slow-expanding lesions with a rim of iron-laden microglia/macrophages. In a recent study, patients with 4 or more iron-rim lesions developed motor and cognitive disabilities at a younger age and were 1.6 times more likely to be diagnosed with progressive MS than those without iron-rim lesions. ${ }^{\text {e44 }}$ These results further strengthen the notion that chronic active lesions play a key role in MS progression. Moreover, the fact that these lesions are present in patients receiving DMTs that target peripherally mediated inflammation strengthens the notion that new treatments need to target directly CNS processes that contribute to progressive MS. ${ }^{\text {e38 }}$

Patients with progressive MS in whom disease activity evidenced by relapses or imaging features of acute inflammatory activity is present should benefit from treatment. However, the results of recent clinical trials in patients with progressive MS demonstrate that targeting only acute peripherally mediated inflammation is not enough to slow disease progression and that, to be effective in progressive MS, DMTs also need to target chronic inflammation in the CNS. Several drugs with a mode of action that specifically targets inflammation compartmentalized to the CNS and/or neurodegeneration are under investigation at various stages of development. Of these, the only one with positive results in progressive MS phase 2 trials is ibudilast, a small molecule able to cross the BBB. Ibudilast slowed the progression of brain atrophy by $48 \%$ compared with placebo in patients with progressive MS (PPMS or SMPS), an effect attributed to a reduction of inflammatory responses in the CNS, given that ibudilast did not prevent the development of new MRI lesions in patients with RRMS. ${ }^{\text {e45 }}$ Bruton tyrosine kinase (BTK) inhibitors (including evobrutinib [NCT04338022, NCT04338061], tolebrutinib [NCT04411641, NCT04458051], and fenebrutinib [NCT04544449]) are of interest as molecules targeting CNS inflammation because of the expression of BTKs by microglia. Studies investigating the efficacy and safety of treatment combination approaches that target acute, peripherally mediated inflammation and chronic inflammation in the CNS independently may be warranted.

Conversion from RRMS to SPMS is a critical event, not only because it implies the inexorable progression of disability, but also because, until recently, relapsing MS treatments showed no efficacy in terms of reducing disability progression in SPMS. To date, no clear clinical, imaging, immunologic, or pathologic criteria exist to determine when RRMS transitions into SPMS. There is a need for tools to support early identification of SPMS. Such tools, together with the use of appropriate treatments, may result in better long-term outcomes for this population.

\section{Acknowledgment}

The authors thank Jenny Charlton and Amy Filby (Oxford PharmaGenesis) for copyediting the manuscript. These services were paid for by Novartis Pharma AG.

\section{Study Funding}

Novartis Pharma AG.

\section{Disclosure}

B.A.C. Cree has received personal compensation for consulting from Alexion, Atara, Autobahn, EMD Serono, Novartis, Sanofi, TG Therapeutics, and Therini; and research support from Genentech. D.L. Arnold has received personal fees from Acorda, Albert Charitable Trust, Biogen, Celgene, Frequency Therapeutics, GeNeuro, MedDay, Merck Serono, Novartis, Roche, SanofiAventis, and Wave Life Sciences; grants from Biogen, Immunotec, and Novartis; and has equity interest in NeuroRx, outside the submitted work. J. Chataway has received support, in the past 3 years, from the Efficacy and Evaluation (EME) Programme, the Health Technology Assessment (HTA) Programme, a partnership between the Medical Research Council (MRS) and the National Institute for Health Research (NIHR), the Rosetrees Trust, the UK MS Society, and the US National MS Society; is supported in part by the National Institute for Health Research, Biomedical Research Centre, University College London Hospitals, UK; has been a local principal investigator for a trial in MS funded by the Canadian MS Society and for commercial trials funded by Actelion, Biogen, Novartis, and Roche; has received an investigator grant from Novartis; and has taken part in advisory boards/consultancy for Azadyne, Biogen, Celgene, MedDay, 
Merck, and Roche. T. Chitnis has received research funding from Novartis, Serono, and Verily; and has participated as a consultant or advisor for Biogen Idec, Novartis, and Sanofi Genzyme. RJ. Fox has received personal fees from Actelion, Biogen, Celgene, EMD Serono, Genentech, Immunic, Novartis, and Teva; grants from Novartis; and other support from Biogen and Novartis (clinical trial contracts). A. Pozo Ramajo has no disclosures to report. N. Murphy was an employee of Novartis Pharma AG at the time of writing. H. Lassmann has received fees for lectures from Merck Serono, Novartis, and Sanofi Aventis; and served as a consultant for Biogen Idec, MedDay, and Roche. Go to Neurology.org/ $\mathrm{N}$ for full disclosures.

\section{Publication History}

Received by Neurology October 16, 2020. Accepted in final form May 13, 2021.

\section{Appendix Authors}

\begin{tabular}{|c|c|c|}
\hline Name & Location & Contribution \\
\hline $\begin{array}{l}\text { Bruce A.C. } \\
\text { Cree, MD, } \\
\text { PhD, MAS }\end{array}$ & $\begin{array}{l}\text { UCSF Weill Institute for } \\
\text { Neurosciences, Department } \\
\text { of Neurology, University of } \\
\text { California San Francisco }\end{array}$ & $\begin{array}{l}\text { Design/conceptualization of } \\
\text { the review; interpretation of } \\
\text { data; drafting/revising the } \\
\text { manuscript for intellectual } \\
\text { content }\end{array}$ \\
\hline
\end{tabular}

Douglas L. NeuroRx Research; Brain Arnold, MD Imaging Centre, Montreal Neurological Institute, McGill University, Montreal, Canada

Design/conceptualization of the review; interpretation of data; drafting/revising the manuscript for intellectual content

\begin{tabular}{ll}
\hline Jeremy & Queen Square Multiple \\
Chataway, & Sclerosis Centre, Department \\
PhD, FRCP & of Neuroinflammation, UCL \\
& Queen Square Institute of \\
& Neurology, Faculty of Brain \\
& Sciences, University College \\
& London; National Institute for \\
& Health Research, University \\
& College London Hospitals, \\
& Biomedical Research Centre, \\
UK
\end{tabular}

\begin{tabular}{|c|c|c|}
\hline $\begin{array}{l}\text { Tanuja } \\
\text { Chitnis, MD }\end{array}$ & $\begin{array}{l}\text { Brigham Multiple Sclerosis } \\
\text { Center, Brigham and } \\
\text { Women's Hospital, Boston, } \\
\text { MA }\end{array}$ & $\begin{array}{l}\text { Design/conceptualization of } \\
\text { the review; interpretation of } \\
\text { data; drafting/revising the } \\
\text { manuscript for intellectual } \\
\text { content }\end{array}$ \\
\hline
\end{tabular}

Robert J. Mellen Center for Multiple

Fox, MD Sclerosis Treatment and Research, Neurological Institute, Cleveland Clinic, $\mathrm{OH}$

Design/conceptualization of the review; interpretation of data; drafting/revising the

manuscript for intellectual content

\begin{tabular}{lll}
\hline $\begin{array}{l}\text { Angela Pozo } \\
\text { Ramajo, } \\
\text { PhD }\end{array}$ & $\begin{array}{l}\text { Oxford PharmaGenesis, } \\
\text { Oxford, UK }\end{array}$ & $\begin{array}{l}\text { Drafting/revising the } \\
\text { manuscript for intellectual } \\
\text { content }\end{array}$ \\
\hline $\begin{array}{l}\text { Niamh } \\
\text { Murphy, } \\
\text { PhD }\end{array}$ & $\begin{array}{l}\text { Novartis Pharma AG, Basel, } \\
\text { Switzerland }\end{array}$ & $\begin{array}{l}\text { Design/conceptualization of } \\
\text { the review; interpretation of } \\
\text { data; drafting/revising the } \\
\text { manuscript for intellectual } \\
\text { content }\end{array}$ \\
& &
\end{tabular}

\begin{tabular}{ll}
\hline Hans & Center for Brain Research, \\
Lassmann, & Medical University of Vienna, \\
MD & Austria
\end{tabular}

\section{References}

1. Lassmann H. Pathogenic mechanisms associated with different clinical courses of multiple sclerosis. Front Immunol. 2018;9:3116.

2. Lassmann H, van Horssen J, Mahad D. Progressive multiple sclerosis: pathology and pathogenesis. Nat Rev Neurol. 2012;8(11):647-656.

3. Kappos L, Bar-Or A, Cree BAC, et al. Siponimod versus placebo in secondary progressive multiple sclerosis (EXPAND): a double-blind, randomised, phase 3 study. Lancet. 2018;391(10127):1263-1273.

4. Novartis Pharmaceuticals Corporation. Siponimod highlights of prescribing information. Accessed January 16, 2020, novartis.us/sites/www.novartis.us/files/mayzent.pdf.

5. Novartis Pharma GmbH. Mayzent summary of product characteristics. Accessed February 5, 2020. ema.europa.eu/en/documents/product-information/mayzentepar-product-information_en.pdf.

6. Novartis Europharm Limited. Extavia summary of product characteristics. Accessed September 11, 2020, ema.europa.eu/en/documents/product-information/extaviaepar-product-information_en.pdf.

7. Bayer AG. Betaferon summary of product characteristics. Accessed September 11, 2020, ema.europa.eu/en/documents/product-information/betaferon-epar-productinformation_en.pdf.

8. Merck Europe B.V. Mavenclad summary of product characteristics. Accessed September 11, 2020, ema.europa.eu/en/documents/product-information/mavencladepar-product-information_en.pdf.

9. Roche Registration GmbH. Ocrevus summary of product characteristics. 2018. Accessed February 19, 2021, ema.europa.eu/en/documents/product-information/ ocrevus-epar-product-information_en.pdf.

10. Correale J, Gaitan MI, Ysrraelit MC, Fiol MP. Progressive multiple sclerosis: from pathogenic mechanisms to treatment. Brain. 2017;140(3):527-546.

11. Bevan RJ, Evans R, Griffiths L, et al. Meningeal inflammation and cortical demyelination in acute multiple sclerosis. Ann Neurol. 2018;84(6):829-842.

12. Lassmann H. Multiple sclerosis: lessons from molecular neuropathology. Exp Neurol. 2014;262(pt A):2-7.

13. Haider L, Simeonidou C, Steinberger G, et al. Multiple sclerosis deep grey matter: the relation between demyelination, neurodegeneration, inflammation and iron. J Neurol Neurosurg Psychiatry. 2014;85(12):1386-1395.

14. Witte ME, Mahad DJ, Lassmann H, van Horssen J. Mitochondrial dysfunction contributes to neurodegeneration in multiple sclerosis. Trends Mol Med. 2014;20(3): 179-187.

15. Azevedo CJ, Cen SY, Khadka S, et al. Thalamic atrophy in multiple sclerosis: a magnetic resonance imaging marker of neurodegeneration throughout disease. Ann Neurol. 2018;83(2):223-234.

16. Mahad DH, Trapp BD, Lassmann H. Pathological mechanisms in progressive multiple sclerosis. Lancet Neurol. 2015;14(2):183-193.

17. Frischer JM, Weigand SD, Guo Y, et al. Clinical and pathological insights into the dynamic nature of the white matter multiple sclerosis plaque. Ann Neurol. 2015;78(5):710-721.

18. Luchetti S, Fransen NL, van Eden CG, Ramaglia V, Mason M, Huitinga I. Progressive multiple sclerosis patients show substantial lesion activity that correlates with clinical disease severity and sex: a retrospective autopsy cohort analysis. Acta Neuropathol. 2018;135(4):511-528.

19. Tavazzi E, Zivadinov R, Dwyer MG, et al. MRI biomarkers of disease progression and conversion to secondary-progressive multiple sclerosis. Expert Rev Neurother. 2020; 20(8):821-834.

20. Pipi E, Nayar S, Gardner DH, Colafrancesco S, Smith C, Barone F. Tertiary lymphoid structures: autoimmunity goes local. Front Immunol. 2018;9(8):1952.

21. Chard DT, Miller DH. What lies beneath grey matter atrophy in multiple sclerosis? Brain. 2016;139(pt 1):7-10.

22. Bergsland N, Horakova D, Dwyer MG, et al. Gray matter atrophy patterns in multiple sclerosis: a 10-year source-based morphometry study. Neuroimage Clin. 2018;17:444-451.

23. Schlaeger R, Papinutto N, Zhu AH, et al. Association between thoracic spinal cord gray matter atrophy and disability in multiple sclerosis. JAMA Neurol. 2015;72(8):897-904.

24. Matthews PM. Chronic inflammation in multiple sclerosis - seeing what was always there. Nat Rev Neurol. 2019;15(10):582-593.

25. Colasanti A, Guo $\mathrm{Q}$, Muhlert $\mathrm{N}$, et al. In vivo assessment of brain white matter inflammation in multiple sclerosis with (18) F-PBR111 PET. J Nucl Med. 2014;55(7): 1112-1118.

26. Rissanen E, Tuisku J, Rokka J, et al. In vivo detection of diffuse inflammation in secondary progressive multiple sclerosis using PET imaging and the radioligand ${ }^{11}$ C-PK11195. J Nucl Med. 2014;55(6):939-944.

27. Lorscheider J, Buzzard K, Jokubaitis V, et al. Defining secondary progressive multiple sclerosis. Brain. 2016;139(pt 9):2395-2405.

28. Lublin FD, Reingold SC, Cohen JA, et al. Defining the clinical course of multiple sclerosis: the 2013 revisions. Neurology. 2014;83(3):278-286.

29. Kantarci OH, Lebrun C, Siva A, et al. Primary progressive multiple sclerosis evolving from radiologically isolated syndrome. Ann Neurol. 2016;79(2):288-294.

30. Ransohoff RM, Hafler DA, Lucchinetti CF. Multiple sclerosis - a quiet revolution. Nat Rev Neurol. 2015;11(3):134-142.

31. Okuda DT, Siva A, Kantarci O, et al. Radiologically isolated syndrome: 5-year risk for an initial clinical event. PLoS One. 2014;9(3):e90509.

32. Lebrun-Frenay C, Kantarci O, Siva A, et al. Radiologically isolated syndrome: 10-year risk estimate of a clinical event. Ann Neurol. 2020;88(2):407-417.

33. Scalfari A, Neuhaus A, Daumer M, Muraro PA, Ebers GC. Onset of secondary progressive phase and long-term evolution of multiple sclerosis. J Neurol Neurosurg Psychiatry. 2014;85(1):67-75. 
34. Kappos L, Kuhle J, Multanen J, et al. Factors influencing long-term outcomes in relapsing-remitting multiple sclerosis: PRISMS-15. J Neurol Neurosurg Psychiatry. 2015;86(11):1202-1207.

35. Traboulsee AL, Cornelisse P, Sandberg-Wollheim M, et al. Prognostic factors for long-term outcomes in relapsing-remitting multiple sclerosis. Mult Scler J Exp Transl Clin. 2016;2:2055217316666406.

36. Zeydan B, Gu X, Atkinson EJ, et al. Cervical spinal cord atrophy: an early marker of progressive MS onset. Neurol Neuroimmunol Neuroinflamm. 2018;5(2):e435.

37. Krieger SC, Sumowski J. New insights into multiple sclerosis clinical course from the topographical model and functional reserve. Neurol Clin. 2018;36(1):13-25.

38. Tremlett H, Yousefi M, Devonshire V, Rieckmann P, Zhao Y. Impact of multiple sclerosis relapses on progression diminishes with time. Neurology. 2009;73(20):1616-1623.

39. Kaminsky AL, Omorou AY, Soudant M, et al. Discontinuation of disease-modifying treatments for multiple sclerosis in patients aged over 50 with disease inactivity. J Neurol. 2020;267(12):3518-3527.

40. Katz Sand I, Krieger S, Farrell C, Miller AE. Diagnostic uncertainty during the transition to secondary progressive multiple sclerosis. Mult Scler. 2014;20(12):1654-1657.

41. Cree BAC, Mares J, Hartung HP. Current therapeutic landscape in multiple sclerosis: an evolving treatment paradigm. Curr Opin Neurol. 2019;32(5):365-377.

42. University of California San Francisco EPIC Study, Cree BAC, Hollenbach JA, Bove $\mathrm{R}$, et al. Silent progression in disease activity-free relapsing multiple sclerosis. Ann Neurol. 2019;85(5):653-666.

43. Kappos L, Wolinsky JS, Giovannoni G, et al. Contribution of relapse-independent progression vs relapse-associated worsening to overall confirmed disability accumulation in typical relapsing multiple sclerosis in a pooled analysis of 2 randomized clinical trials. JAMA Neurol. 2020;77(9):1132-1140.

44. Sanai SA, Saini V, Benedict RH, et al. Aging and multiple sclerosis. Mult Scler. 2016; 22(6):717-725

45. Mao Z, Alvarez-Gonzalez C, Allen-Philbey K, et al. Treating the ineligible: disease modification in people with multiple sclerosis beyond NHS England commissioning policies. Mult Scler Relat Disord. 2019;27:247-253.

46. Benedict RH, DeLuca J, Phillips G, et al. Validity of the Symbol Digit Modalities Test as a cognition performance outcome measure for multiple sclerosis. Mult Scler. 2017;23(5):721-733.

47. Zhang J, Waubant E, Cutter G, Wolinsky J, Leppert D. Composite end points to assess delay of disability progression by MS treatments. Mult Scler. 2014;20(11):1494-1501.
48. Romero K, Shammi P, Feinstein A. Neurologists accuracy in predicting cognitive impairment in multiple sclerosis. Mult Scler Relat Disord. 2015;4(4):291-295.

49. Sumowski JF, Benedict R, Enzinger C, et al. Cognition in multiple sclerosis. Neurology. 2018;90(6):278

50. Corfield F, Langdon D. A systematic review and meta-analysis of the Brief Cognitive Assessment for Multiple Sclerosis (BICAMS). Neurol Ther. 2018;7(2):287-306.

51. Marziniak M, Brichetto G, Feys P, Meyding-Lamade U, Vernon K, Meuth SG. The use of digital and remote communication technologies as a tool for multiple sclerosis management: narrative review. JMIR Rehabil Assist Technol. 2018;5(1):e5.

52. Block VJ, Bove R, Zhao C, et al. Association of continuous assessment of step count by remote monitoring with disability progression among adults with multiple sclerosis. JAMA Netw Open. 2019;2(3):e190570.

53. Barbour C, Kosa P, Komori M, et al. Molecular-based diagnosis of multiple sclerosis and its progressive stage. Ann Neurol. 2017;82(5):795-812.

54. Ziemssen $\mathrm{T}$, Tolley $\mathrm{C}$, Bennett $\mathrm{B}$, et al. A mixed methods approach towards understanding key disease characteristics associated with the progression from RRMS to SPMS: physicians' and patients' views. Mult Scler Relat Disord. 2019;38:101861.

55. Miller DH, Lublin FD, Sormani MP, et al. Brain atrophy and disability worsening in primary progressive multiple sclerosis: insights from the INFORMS study. Ann Clin Transl Neurol. 2018;5(3):346-356.

56. Mulero P, Midaglia L, Montalban X. Ocrelizumab: a new milestone in multiple sclerosis therapy. Ther Adv Neurol Disord. 2018;11:1756286418773025.

57. Genentech Inc. Ocrevus highlights of prescribing information. Accessed January 16, 2020, gene.com/download/pdf/ocrevus_prescribing.pdf.

58. European Medicines Agency (EMA) press release. New medicine for multiple sclerosis. Accessed January 16, 2020. ema.europa.eu/en/news/new-medicine-multiplesclerosis.

59. Cree BAC, Cutter G, Wolinsky J, et al. Results of the phase 3 SPI2 study of MD1003 (high dose pharmaceutical grade biotin) in progressive MS. AAN 2020 Science Highlights. 2020. Accessed September 14, 2020, cslide-us.ctimeetingtech.com/ aan2020/attendee/eposter/poster/2138? q=cree

60. Lublin F, Miller DH, Freedman MS, et al. Oral fingolimod in primary progressive multiple sclerosis (INFORMS): a phase 3, randomised, double-blind, placebocontrolled trial. Lancet. 2016;387(10023):1075-1084.

eReferences e1-e45 are available at Dryad (doi.org/10.5061/dryad.msbcc2fxn).

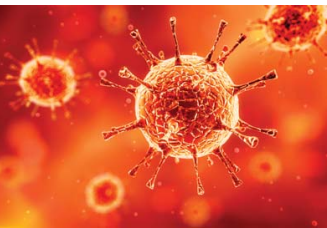

\section{COVID-19 and Neurologic Disease: Call for Papers!}

The editors of Neurology are interested in papers that address the neurological aspects of COVID-19 infection and challenges to the management of patients with chronic neurological conditions who have, or are at risk for, the infection. Relevant papers that pass initial internal review will undergo expedited peer review and online publication. We will consider papers posted in preprint servers.

Submit observational studies and clinical trials as Articles and case series and case reports under the Clinical/Scientific Notes category to https://submit.neurology.org/ today!

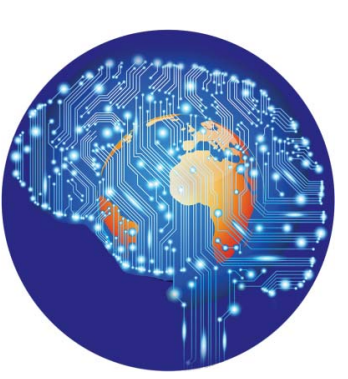

\section{Practice Current: An interactive exchange on controversial topics}

Share your own best practices.

Read commentary with expert opinion.

Explore results on an interactive world map.

NPub.org/NCP/practicecurrent

Neurology ${ }^{\circledR}$ Clinical Practice 


\title{
Neurology
}

\author{
Secondary Progressive Multiple Sclerosis: New Insights \\ Bruce A.C. Cree, Douglas L. Arnold, Jeremy Chataway, et al. \\ Neurology 2021;97;378-388 Published Online before print June 4, 2021 \\ DOI 10.1212/WNL.0000000000012323
}

This information is current as of June 4, 2021

\section{Updated Information \& Services}

\section{References}

Citations

Subspecialty Collections

Permissions \& Licensing

Reprints including high resolution figures, can be found at: http://n.neurology.org/content/97/8/378.full

This article cites 51 articles, 8 of which you can access for free at: http://n.neurology.org/content/97/8/378.full\#ref-list-1

This article has been cited by 2 HighWire-hosted articles: http://n.neurology.org/content/97/8/378.full\#\#otherarticles

This article, along with others on similar topics, appears in the following collection(s):

Autoimmune diseases

http://n.neurology.org/cgi/collection/autoimmune_diseases

Multiple sclerosis

http://n.neurology.org/cgi/collection/multiple_sclerosis

Information about reproducing this article in parts (figures,tables) or in its entirety can be found online at:

http://www.neurology.org/about/about_the_journal\#permissions

Information about ordering reprints can be found online:

http://n.neurology.org/subscribers/advertise

Neurology ${ }^{\circledR}$ is the official journal of the American Academy of Neurology. Published continuously since 1951, it is now a weekly with 48 issues per year. Copyright Copyright @ 2021 The Author(s). Published by Wolters Kluwer Health, Inc. on behalf of the American Academy of Neurology.. All rights reserved. Print ISSN: 0028-3878. Online ISSN: 1526-632X.

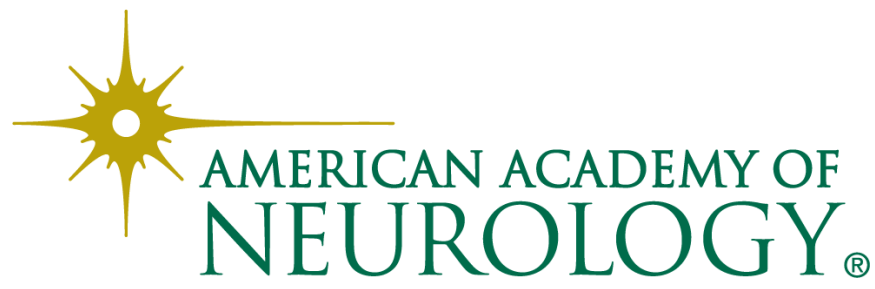

